\title{
Lymphohematopoietic progenitors immortalized by a retroviral vector harboring a dominant-negative retinoic acid receptor can recapitulate lymphoid, myeloid, and erythroid development
}

\author{
Schickwann Tsai, ${ }^{1}$ Stephen Bartelmez, ${ }^{2}$ Ewa Sitnicka, ${ }^{2}$ and Steven Collins \\ Fred Hutchinson Cancer Research Center, Seattle, Washington 98104 USA; ${ }^{2}$ Pathology Department, University of \\ Washington, Seattle, Washington 98195 USA
}

\begin{abstract}
The lymphohematopoietic progenitors represent $<0.01 \%$ of nucleated marrow cells. Here, we describe the immortalization of the murine lymphohematopoietic progenitors by a retroviral vector harboring a dominant-negative retinoic acid receptor. The immortalized progenitors proliferate as a stem-cell-factor-dependent clonal line EML that spontaneously generates pre-pro-B lymphocytes and erythroid and myeloid progenitors. Upon stimulation with interleukin-7 and stromal cells, the pre-pro-B lymphocytes express $R A G-1$ and undergo $D-J$ rearrangements of the immunoglobulin heavy-chain genes. With erythropoietin the erythroid progenitors proliferate and differentiate into red cells. Generation of the common progenitors for neutrophils and macrophages is suppressed in EML but is inducible by high concentrations of retinoic acid. An additional block in neutrophil differentiation occurs at the promyelocyte stage but can also be overcome by high concentrations of retinoic acid. These studies demonstrate a reproducible way to immortalize lymphohematopoietic progenitors and implicate specific roles for retinoic acid receptors at two distinct stages of hematopoiesis.
\end{abstract}

[Key Words: Retinoic acid receptors; lymphohematopoietic progenitors; B-cell development; myelopoiesis; erythropoiesis]

Received August 30, 1994; revised version accepted October 13, 1994.

The existence of a common progenitor that gives rise to all lymphoid and hematopoietic (or "myeloerythroid") cells was initially demonstrated by transplantation experiments using bone marrow cells carrying X-ray-induced chromosomal markers (Wu et al. 1968). More recently, direct marking of bone marrow cells with retroviral vectors has further comfirmed the existence of a common lymphohematopoietic progenitor cell (Lemischka et al. 1986). Through a combination of physical and immunological purification methods, the murine lymphohematopoietic stem cells have been purified to near homogeneity (Spangrude et al. 1988). Their frequency is estimated to be $0.01-0.005 \%$ of all nucleated cells in the bone marrow. The scarcity of the lymphohematopoietic stem cells and the difficulty in their purification and maintenance have hampered the effort to dissect the molecular mechanism controlling lymphoid and hematopoietic development. Thus, the availability of a continuous cell line capable of both lymphoid and mye-

${ }^{1}$ Corresponding author. loerythroid differentiation in vitro would greatly facilitate such research.

In this paper we demonstrate that the lymphohematopoietic progenitors can be immortalized by a retroviral vector containing a dominant-negative retinoic acid receptor construct and that the resulting lymphohematopoietic progenitor cell line exhibits two developmental defects in the myeloid lineage that can be corrected with high concentrations of retinoic acid (RA). The RARs are members of the steroid/thyroid hormone receptors that function as ligand-inducible transcription factors (Evans 1988). Clues suggesting that RARs are involved in regulating hematopoiesis come from both laboratory and clinical observations; RA induces the HL-60 human leukemia cell line to differentiate into mature neutrophils, and this process is mediated through RARs (Breitman et al. 1980; Collins et al. 1990). In most cases of acute promyelocytic leukemia (APL), the gene of RAR $\alpha$ on chromosome 17 is translocated and fused with the PML gene on chromosome 15 (Alcalay et al. 1990; Borrow et al. 1990; de The et al. 1990), and the leukemic cells from these patients can be induced by RA to differ- 
entiate into mature neutrophils both in vitro and in vivo (Huang et al. 1988). The precise role of the PML-RAR $\alpha$ fusion gene in the pathogenesis of APL is yet to be resolved. Attempts to demonstrate a dominant-negative function for $P M L-R A R \alpha$ fusion gene using transient expression assay systems yielded inconsistent results (Kakizuka et al. 1991; de The et al. 1991). On the other hand, we have shown that the expression of a dominant-negative $\operatorname{RAR} \alpha(\operatorname{RAR} \alpha 403)$ in normal mouse bone marrow cells leads to a differentiation block in the neutrophil lineage at the promyelocyte stage (Tsai and Collins 1993). This finding provides direct evidence that RARs play important roles at the promyelocyte stage of neutrophil differentiation and raises the possibility that a novel dominant-negative mechanism may underlie the action of the PML-RAR $\alpha$ fusion protein (Dyck et al. 1994; Weis et al. 1994). We have also shown that when the dominant-negative RAR $\alpha 403$ is expressed in a multipotent, interleukin-3 (IL-3)-dependent myeloid cell line FDCP mix A4, it triggers a rapid switch from spontaneous neutrophil and macrophage development to the production of mast cells (Tsai et al. 1992). This developmental switch appears to occur near or at the lineage decision stage rather than at the neutrophilic promyelocyte stage. Taken together, these observations indicate that RARs not only play important roles in controlling the terminal differentiation of neutrophilic promyelocytes but also may influence hematopoietic lineage development at the multipotent progenitor stage.

To investigate the developmental effects of the dominant-negative RAR $\alpha 403$ on normal, primitive lymphohematopoietic progenitors, we used retroviral vectormediated gene transfer to tranduce fresh mouse bone marrow cells with this dominant-negative RAR and cultured the cells in a medium containing hematopoietic growth factors that promote the survival and proliferation of primitive lymphohematopoietic progenitors. Using this approach we have reproducibly established continuous cell lines that are dependent on stem cell factor (SCF; also known as c-kit ligand) (Zsebo et al. 1990) for survival and proliferation yet can undergo orderly lymphoid, myeloid, and erythroid differentiation in vitro. In addition to demonstrating a reproducible way to immortalize lymphohematopoietic progenitors, our present findings also provide the first evidence that RA and RARs may modulate myeloid lineage development prior to the stage of the so-called colony-forming unit-granulocyte/macrophage (CFU-GM). Further observations suggest that there may be a causal link between the suppression of myeloid development and the immortalization of these lymphohematopoietic progenitors.

\section{Results}

Infection of mouse bone marrow with a retroviral vector LRAR $\alpha 403 S N$ and the establishment of a stem cell factor-dependent cell line

We have described previously the construction of the retroviral vector LRAR $\alpha 403$ SN harboring a truncated
RAR $\alpha$ cDNA insert (Tsai et al. 1992). The truncated $\operatorname{RAR} \alpha$, designated $\operatorname{RAR} \alpha 403$, contains the first 403 amino acids of human RAR $\alpha$ but is truncated in the ligand-binding domain and the activation function domain-1 (AF-1; Nagpal et al. 1992). It exhibits strong dominant-negative activity against endogenous RARs in transient expression assays in 3T3 fibroblasts, CV-1 cells, and hematopoietic cells (Tsai et al. 1992; Damm et al. 1993; Tsai and Collins 1993).

Bone marrow cells $\left(10^{5}\right)$ of male $\mathrm{BDF}_{1}$ mice who received 5-fluorouracil 5 days earlier were infected with the retroviral vector LRAR $\alpha 403 \mathrm{SN}$ and subsequently cultured in a medium containing SCF, IL-3, and erythropoietin (Epo) as detailed in Materials and methods. No G418 selection was applied. An SCF-dependent cell line, designated EML, emerged from this culture. It is nonadherent and has been in continuous passage for $>1$ year with a doubling time of 18-20 hr. In the absence of SCF, the majority (>95\%) of the EML cells die within $24 \mathrm{hr}$, with the rest dying during the next $72 \mathrm{hr}$. It harbors the LRAR $\alpha 403 S N$ provirus genome (Fig. 1A) and expresses high levels of the retroviral message encoding the dominant-negative RAR (Fig. 1B). Many cells (20-30\%) in the EML line have a characteristic hand-mirror shape (Fig. 6A, below).

To ensure the clonality of the cell line, it was first cloned by limiting dilution and recloned at a low cell density in a methylcellulose culture medium as detailed in Materials and methods. Southern analysis reveals that all clones have a single, identical integration site (Fig. 1A), indicating that they are all clonal derivatives of the same parental cell. Importantly, the clonal line and its subclones that we have examined possess similar lineage repertoires described below, although only the data obtained with EML $\mathrm{Cl}$ and its subclone EML C1.4 will be presented in this paper.

\section{The dominant-negative RAR $\alpha 403$ is essential for establishing the EML cell line}

To determine whether the dominant-negative RAR $\alpha 403$ plays an essential role in the establishment of the EML cell line, we repeated the infection of post-5-fluorouracil mouse bone marrow with the control vector LXSN as well as LRAR $\alpha 403 S N$ and processed the cells in parallel. An uninfected bone marrow culture was also included as an additional control. To increase the number of lymphohematopoietic progenitors as potential targets for retroviral infection, $1,000,000$ bone marrow mononuclear cells (rather than $10^{5}$ as in the initial isolation of the EML cell line) were used. Using this approach we have reproducibly isolated EML-like cell lines from mouse bone marrow cells infected with the LRAR $\alpha 403 S N$ vector. In contrast, no cell lines could be isolated from parallel cultures of uninfected or LXSN (control)-infected mouse bone marrow cells. Thus, the dominant-negative RAR $\alpha 403$ is essential for the establishment of the EML cell line. Southern analysis of the proviral integration sites revealed the presence of multiple clones on day 76 after retroviral infection, all of 


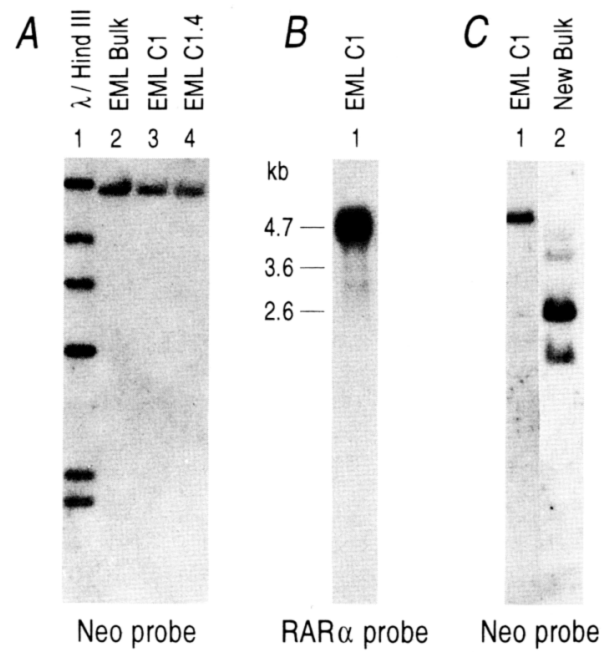

Figure 1. Integration and expression of the LRAR $\alpha 403$ SN provirus in the EML line. (A) Southern analysis of the proviral integration sites. Genomic DNAs were digested with EcoRI (which cuts only once within the provirus upstream of the neo sequencel, blotted, and hybridized with a neo probe. (Lane 1) Uncloned EML line that has been passaged for $2 \frac{1}{2}$ months since retroviral infection and is already dominated by one clone; (lane 2) a clonal line (EML C1) obtained by limiting dilution cloning, followed by recloning in semisolid medium; (lane 3) EML Cl.4 recloned again from EML $\mathrm{Cl}$ in semisolid medium. All clones share the same, single proviral integration site. Comparison of the signal intensity on Southern blot with that of a reference cell line indicates that there is one copy of the provirus per genome. (B) Northern analysis of the expression of the RAR $\alpha 403$ in EML. Five micrograms of total RNA of EML C1 was analyzed using a human RAR $\alpha$ probe. The $4.7-\mathrm{kb}$ retroviral message harboring the RAR $\alpha 403$ sequence and the 3.6- and 2.6$\mathrm{kb}$ endogenous mouse RAR $\alpha$ mRNAs are indicated. $(C)$ Multiclonal origin of EML-like cell lines. Additional EML-like cell lines were established as described for the original EML cell line except that $1,000,000$ rather than $10^{5}$ bone marrow mononuclear cells were used in retroviral infection. Genomic DNA was obtained from a bulk culture 76 days after retroviral infection, digested with EcoRI, and subjected to Southern analysis. (Lane 1) EML Cl; (lane 2) day 76 bulk culture of EML-like cell lines from an independent infection experiment. Four dominant clones are visible and show different integration sites from EML $\mathrm{Cl}$.

which integrated at different sites from EML C1 (Fig. $1 \mathrm{Cl}$, indicating that a common retroviral integration site is probably not required for the immortalization of EMLlike cells. The lymphohematopoietic potential of these new clones was verified after subcloning (not shown). Despite the fact that no G418 selection was applied throughout the experiment, all independently isolated EML-like cell lines we have examined harbored the LRAR $\alpha 403 S N$ provirus, albeit at different integration sites.

The EML cell line contains cells expressing B-lymphocyte-specific antigen $B 220$

The cell surface antigen profile of a representative clone
(C1) of the EML cell line is examined by flow cytometry using a panel of monoclonal antibodies: (1) anti-Sca-1, which is specific for stem cells/primitive progenitors (Spangrude et al. 1988); (2) anti-B220 (clone RA3-6B2), which is specific for the B-lymphocyte lineage /Coffman and Weissman 1981); (3) anti-Mac-1, which is specific for macrophage/neutrophil lineages; (4) monoclonal antibody " $7 / 4$," which is specific for the neutrophil lineage (Hirsch and Gordon 1983); and (5) monoclonal antibody "ter 119," which recognizes erythroid precursors (Ikuta et al. 1990).

As shown in Figure 2, 50\% of EML Cl cells are positive for Sca-1, $\sim 53 \%$ express B220, $\sim 51 \%$ express ter 119 , very few express $7 / 4$ or Mac-1. A similar surface antigen profile is observed in subclones C1.1, C1.3, and C1.4 of EML, although the actual percentages vary (not shown). The B220 antigen, a member of the "leukocyte common antigen" (LCA) family, is specific for primitive as well as mature B cells (for review, see Thomas 1989). Thus, the results of flow cytometry indicate that cells of B-lymphoid and erythroid lineages are spontaneously generated in the EML C1 cell line.

\section{The B-cell progenitors in the EMLC1 cell line are pre-pro-B cells}

To determine the developmental stage of the putative B-cell progenitors present in the EML C1 line, we examined (1) the expression of immunoglobulin heavy chain $(\mathrm{IgH})$ and the primitive lymphocyte-specific gene $R A G-1$ (necombination-activating gene- $\underline{1}$; Schatz et al. 1989) by Northern analyses, and (2) the rearrangement of $D-I$ segments of the IgH gene by polymerase chain reaction (PCR) amplification of genomic DNAs using specific oligonucleotide primers.

When the EML $\mathrm{Cl}$ cell line was maintained in me-

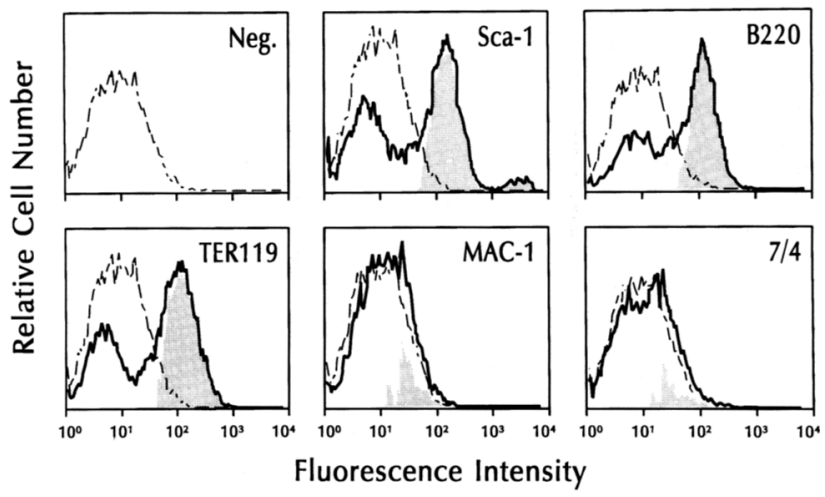

Figure 2. Flow cytometry analysis of the cell surface antigens of EML C1. The profile of the isotype antibody (negative control; thin line/ is shown in the first panel and again in all other panels for direct comparison. The antibodies used and the positive fractions (shaded areas) are indicated in each panel. The specificity of the monoclonal antibodies are as follows: stem cells/primitive progenitors (Sca-1); B cells (B220); neutrophils (7/4); macrophages and neutrophils (Mac-1); erythroid precursors (ter 119). 
dium containing SCF alone, we could detect the expression of a $2.1-\mathrm{kb}$ mRNA and, to a lesser extent, a $3.0-\mathrm{kb}$ mRNA corresponding to the $\mathrm{Mu} 0$ and $\mathrm{I} \mu$ transcripts of the unrearranged germ-line heavy-chain genes (Fig. 3A, lane 4). The 2.1-kb Mu0 transcript is initiated from a promoter upstream of the last D (diversity) segment gene, whereas the $3.0-\mathrm{kb} I \mu$ transcript is initiated from a promoter situated between the J (joint) segment gene and the first exon of the IgH constant region gene (Nelson et al. 1983; Schlissel et al. 1991). This transcription of germ-line $\mathrm{IgH}$ gene precedes or coincides with rearrangement of the gene in developing B lymphocytes and has

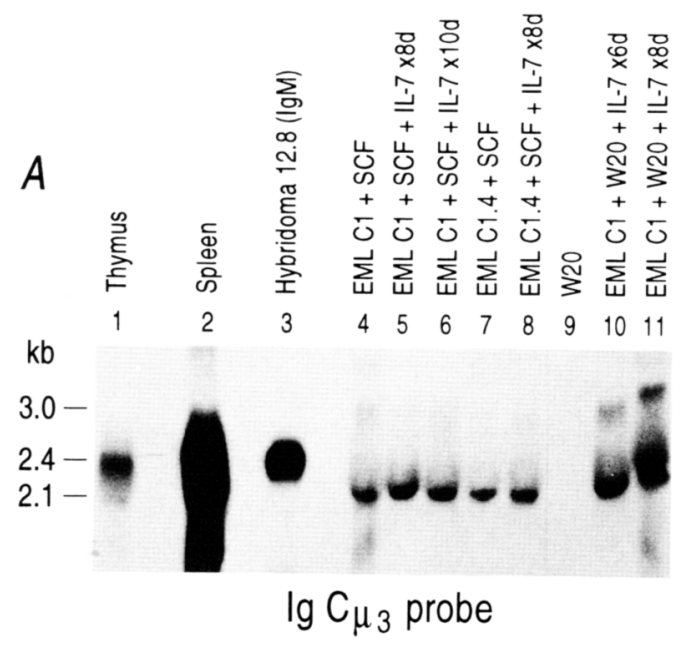

$B$

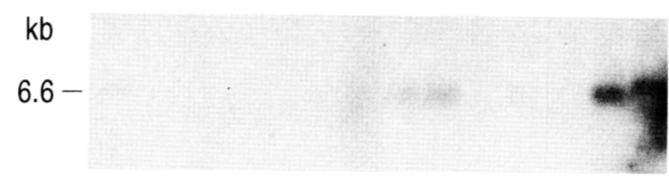

RAG-1 probe

Figure 3. Northern analyses of the expression of $R A G-1$ and immunoglobulin heavy-chain ( $\mathrm{IgH}$ ) genes in EML. Twenty-two micrograms (unless indicated otherwise) of total RNAs were loaded in each lane. (Lane 1) Thymus of an 8-week-old mouse; (lane 2) spleen $(1 \mu \mathrm{g}) ;$ (lane 3) an IgM-secreting hybridoma 12.8 $(1 \mu \mathrm{g}$ ); (lane 4) EML C1 in SCF alone; (lanes 5-6) EML Cl in SCF plus IL-7 for 8 and 10 days, respectively; (lane 7) EML Cl.4 in SCF alone; (lane 8) EML C1.4 in SCF plus IL-7 for 8 days; (lane 9) the stromal cell line W20; (lanes 10-11) EML C1 plus W20 plus IL-7 for 6 and 8 days, respectively. $(A)$ The blot was hybridized with the third exon (immunoglobulin $\mathrm{C}_{\mu 3}$ ) of the mouse IgH constant region. Note the expression and the relative positions of the $2.1-\mathrm{kb} \mathrm{MuO}$ and the $3.0-\mathrm{kb} \mathrm{I} \mu$ germ-line IgH transcripts in EML. The position of the $2.4-\mathrm{kb}$ secretory IgM heavychain mRNA of hybridoma 12.8 is also indicated for comparison. The seemingly slower migration in lane 11 is a gel artifact. (B) Expression of RAG-1. A 0.95-kb RAG-1 genomic DNA was used to probe the same blot as $A$. Note the high-level expression of the 6.6-kb RAG-1 mRNA by EML cells cocultured with W20 and IL-7 compared with thymus and EML cells stimulated with SCF and IL-7 alone. Film was exposed for 5 days. long been hypothesized to be a prerequisite for immunoglobulin gene rearrangement (Alt et al. 1987; Schlissel and Baltimore 1989). PCR of genomic DNA from SCFdependent EML $\mathrm{C} 1$ cells showed no significant $D-I$ rearrangement (Fig. 4B, lane 3). Thus, our findings of germline heavy-chain transcripts without significant $D-I$ rearrangement suggest that the B220-positive cells in the EML C1 line are B-cell progenitors at a very early stage of lymphopoiesis, that is, the pre-pro-B stage (Hardy et al. 1991). Not surprisingly, no cytoplasmic or surface $\mu$ chain could be detected by direct immunofluorescent staining of EML C1 cells.

A bone marrow stromal cell line, together with $I L-7$, stimulates EML C1-derived B-cell progenitors to proliferate and to initiate $\mathrm{D}-\mathrm{J}$ rearrangement

Because the combination of bone marrow stromal cells and IL-7 has been shown to support the proliferation and differentiation of early B cells (Kierney and Dorshkind 1987), the EML Cl cells were cocultured with bone marrow stromal cell line W20 (Tsai 1986) and IL-7 but without added SCF. IL-7 $(100 \mathrm{U} / \mathrm{ml})$ alone did not support the survival of EML C1 cells, whereas W20 alone allowed limited proliferation. In contrast, the combination of W20 and IL-7 $(100 \mathrm{U} / \mathrm{ml})$ triggered rapid proliferation of EML Cl-derived cells. By indirect immunofluorescence microscopy, the proliferating cells were B220-positive and remained attached to the stromal cells (Fig. 6E, below). The requirement of both stromal cells and IL-7 for proliferation is regarded as a characterisitic of early B-cell progenitors (Kierney and Dorshkind 1987). Southern analysis revealed that the B-cell precursors that proliferated in response to W20 plus IL-7 shared the same proviral integration site as EML $\mathrm{Cl}$ (Fig. 7B, below). Northern analyses of the EML C1/W20/IL-7 cocultures showed induction of RAG-1 mRNA after 4-6 days (Fig. $3 \mathrm{~B}$, lane 10). PCR analyses of genomic DNAs of EML $\mathrm{C} 1 / \mathrm{W} 20 / \mathrm{IL}-7$ cocultures showed significant $D-I$ rearrangements after $6-8$ days of coculture (Fig. $4 \mathrm{~B}$, lanes $5,6)$. The timing of $D-I$ rearrangement correlates well with the temporal expression of the RAG-1 gene that is thought to encode a putative recombinase involved in the rearrangements of the immunoglobulin and T-cell receptor genes (Schatz et al. 1989). In contrast, EML C1 cells stimulated with SCF and IL-7 alone (but without stromal cells) for 8 and 10 days revealed comparatively smaller increases in RAG-1 expression (Fig. 3B, lanes 5,6) and $D-I$ rearrangements (not shown).

\section{Erythroid progenitors are generated in the EML C1} cell line

Approximately $50 \%$ of the EML Cl cells stained positive with monoclonal antibody ter 119 (Fig. 2), suggesting that there were erythroid progenitors in the EML C1 line. Within 4 days after Epo (plus SCF) was added to the suspension culture of EML CI cells, many erythroblasts at all stages of hemoglobinization appeared as clusters of $4-32$ cells consitituting $\sim 20 \%$ of all cells (Figs. 5A and 


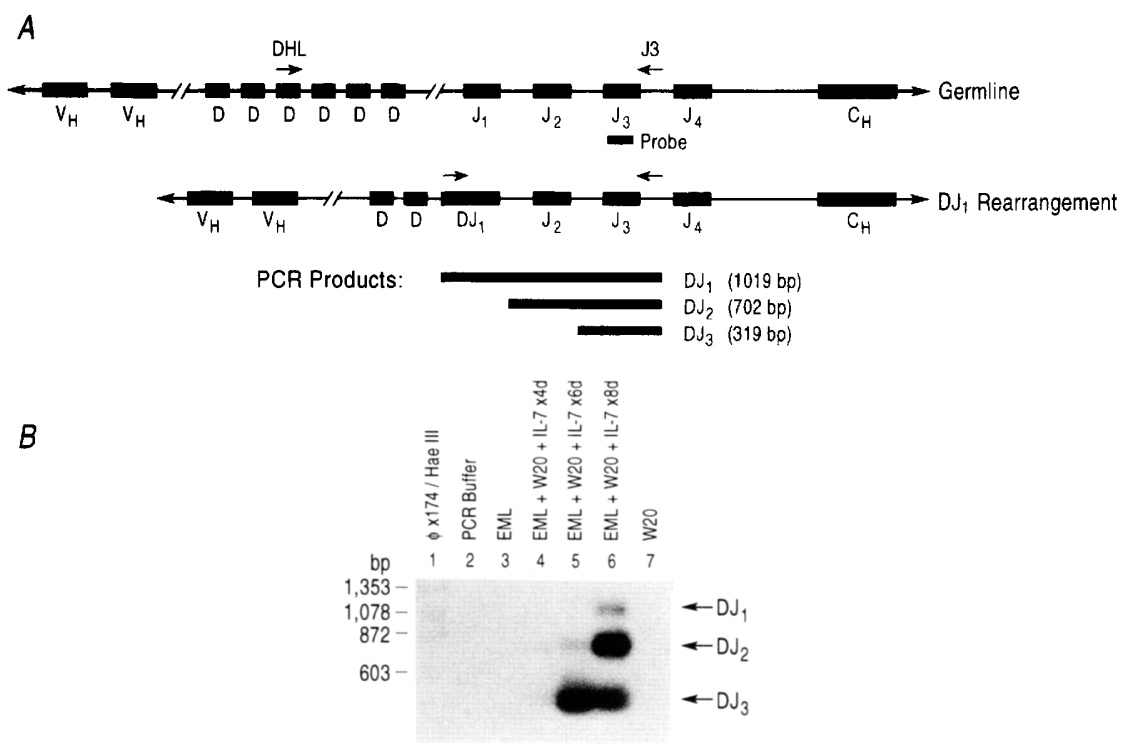

Figure 4. PCR detection of $D-J$ rearrangements in EML Cl-derived B-cell progenitors. $(A)$ The locations of the DHL and J3 primers and the 226-bp $I_{3}$ Southern probe in $\mathrm{IgH}$ gene are depicted. The sizes $(\sim 319$, $\sim 702$, and $\sim 1019 \mathrm{bp}$ ) of various amplification products are approximate estimates. (B) $D-I$ rearrangements detected by PCR of genomic DNAs. The DHL and $\mathrm{J} 3$ primers were used in PCR. The Southern blot of PCR products was hybridized with the 226bp $I_{3}$ probe. (Lane 1) $\Phi \mathrm{X} 174 /$ HaeIII digests as size markers; (lane 2) PCR buffer; (lane 3) EML Cl in SCF; (lanes 4-6) EML Cl cocultured with stromal cells W20 plus IL-7 for 4,6 , and 8 days, respectively; (lane 7) stromal cell line W20.

6B, below). The level of globin mRNA in these SCF/Epotreated EML $\mathrm{Cl}$ cells could approach that of hexamethylene bisacetamide (HMBA)-induced murine erythroleukemia cell line (MEL) (Fig. 5B). When EML C1 cells were cultured in methylcellulose medium containing SCF and Epo, typical burst-forming unit-erythroid (BFU-E)-derived colonies containing thousands of erythroblasts as well as mixed colonies composed of both undifferentiated EML $\mathrm{Cl}$ cells and BFU-E-derived subcolonies were observed. The frequency of BFU-Es is $\sim 1-2 \%$ of the total population in EML $\mathrm{Cl}$. These observations indicate that the SCF-dependent EML Cl cells spontaneously generate erythroid progenitors that terminally differentiate in response to Epo.
$A$

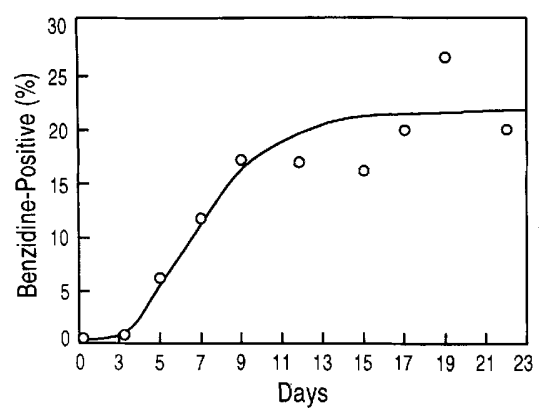

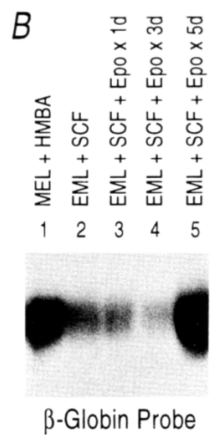

B-Globin Probe
Figure 5. Erythroid differentiation of EML Cl. (A) Time course of the appearance of benzidine (hemoglobin)-positive erythroblasts after addition of Epo. Numbers are means of duplicates. (B) Northern analysis of the expression of mouse $\beta^{\text {major }}$ globin mRNA. (Lane 1) HMBA-induced MEL cell line; (lanes 2-5) EML $\mathrm{Cl}$ cells stimulated with both SCF $(200 \mathrm{ng} / \mathrm{ml})$ and Epo $(8$ $\mathrm{U} / \mathrm{ml}$ ) for $0,1,3$, and 5 days, respectively. The lower message level in lane 4 is the result of the suboptimal culture condition caused by a higher cell density.

\section{Induction of myeloid progenitors (CFU-GMs) from EML C1 cells}

Few EML C1 cells express neutrophil or macrophage lineage-specific surface markers (Fig. 2), suggesting that very few myeloid cells or their progenitors are generated in EML C1 cells maintained in SCF alone. Colony assays of CFU-GMs using murine granulocyte/macrophage colony-stimulating factor (GM-CSF) detected very few or no CFU-GMs in EML Cl cells (Table 1). Costimulation of EML Cl with both SCF and IL-3 resulted in some increase in CFU-GMs. More importantly, simultaneous treatment of EML Cl cells with SCF, IL-3, and high concentrations of RA $\left(0.5-1 \times 10^{-5} \mathrm{M}\right)$ dramatically increased the generation of CFU-GMs (Table 1), which formed colonies of neutrophils, macrophages, and promyelocytes in semisolid medium in the presence of GMCSF. This induction of CFU-GMs by RA has never been described before. Interestingly, the combination of SCF, GM-CSF, and $10^{-5} \mathrm{M}$ RA did not induce the formation of CFU-GMs (not shown). This is consistent with the observation that multiple growth factors such as IL-3 and GM-CSF applied sequentially are needed for successful generation of CFU-GMs from stem cells (Metcalf 1993). The optimal concentration of RA for induction of CFUGMs from EML Cl cells was $10^{-5} \mathrm{M}$ with the half-optimal concentration being $10^{-6} \mathrm{M}$. The induction of CFUGMs by RA (in the presence of SCF and IL-3) could be detected as early as $24 \mathrm{hr}$ after adding RA. This dramatic increase in CFU-GMs was accompanied by a reduction in the numbers of BFU-Es and of self-renewing clonogenic cells in SCF/IL-3/RA-treated EML Cl cultures when compared with cultures treated with SCF/IL-3 alone (Table 1). These results suggest that the formation of CFU-GMs is severely suppressed in the EML Cl cells, probably by the dominant-negative $\operatorname{RAR} \alpha 403$, and that this suppression can be overcome with high concentrations of RA in presence of IL-3. 
Table 1. Effects of RA on the production of CFU-GM, $B F U-E$, and the self-renewal in EML C1

\begin{tabular}{|c|c|c|c|c|}
\hline SCF & + & + & + & + \\
\hline IL-3 & - & - & + & + \\
\hline $\mathrm{RA}\left(10^{-5} \mathrm{M}\right)$ & - & + & - & + \\
\hline \multicolumn{5}{|l|}{$\begin{array}{r}\text { Experiment } 1(n=2) \\
\text { total cells on day } 3\end{array}$} \\
\hline$\left(\times 10^{6}\right)$ & 2.0 & 0.6 & 3.9 & 3.1 \\
\hline total CFU-GMa & 2.5 & 0 & 1,115 & 32,040 \\
\hline $\begin{array}{l}\text { total SCF-supported } \\
\text { clonogenic cells }{ }^{\mathrm{b}}\end{array}$ & 5,200 & 1,000 & 7,400 & 2,000 \\
\hline \multicolumn{5}{|l|}{$\begin{array}{l}\text { Experiment } 2(n=3) \\
\text { total cells on day } 4\end{array}$} \\
\hline$\left(\times 10^{6}\right)$ & 7.0 & N.D. & 17.4 & 13.3 \\
\hline total CFU-GM & 0 & N.D. & 150 & 12,850 \\
\hline $\begin{array}{l}\text { total SCF-supported } \\
\text { clonogenic cells }\end{array}$ & 58,600 & N.D. & 68,600 & 8,600 \\
\hline total BFU-E ${ }^{c}$ & 182,800 & N.D. & 276,000 & 42,800 \\
\hline
\end{tabular}

EML C1 cells $\left(10^{5}\right)$ were cultured in a liquid medium containing $\mathrm{SCF} \pm \mathrm{IL}-3 \pm \mathrm{RA}$ for 3 days (experiment 1 ) or 4 days (experiment 2). Cells were then washed with PBS and counted, and aliquots were cultured in methylcellulose medium to assay for various types of progenitors. Higher-passage cells were used in experiment 2.

${ }^{a}$ For CFU-GM assay, the methylcellulose medium was supplemented with murine GM-CSF $(10 \mathrm{ng} / \mathrm{ml})$.

${ }^{b}$ For SCF-supported clonogenic cells, i.e., the self-renewing cells, the methylcellulose medium was supplemented with rat SCF $(200 \mathrm{ng} / \mathrm{ml})$.

'For BFU-E assay, the methylcellulose medium was supplemented with rat SCF $(200 \mathrm{ng} / \mathrm{ml})$ and Epo $(4 \mathrm{U} / \mathrm{ml})$. Only pure BFU-E colonies were counted.

\section{Other lineages detected in the EML C1 cell line}

When EML C1 cells were stimulated with IL-3 (which is essential for mast cell differentiation and survival) in liquid culture with or without SCF, we observed the appearance of mast cells (Fig. 6C). With simultaneous stimulation with SCF, IL-3, Epo, IL-6, and IL-11, occasional megakaryocytes were detected (Fig. 6D). When multiple growth factors such as SCF, IL-3, GM-CSF, and Epo were added to colony assays of EML Cl cells, we could readily detect multilineage colonies. Taken together, our data indicate that the SCF-dependent EML Cl cell line contains progenitors capable of differentiation along B-lymphocyte, erythrocyte, neutrophil, macrophage, mast cell, and megakaryocyte lineages.

The terminal differentiation of EML C1-derived neutrophil precursors is further blocked at the promyelocyte stage by the dominant-negative RAR $\alpha 403$

When EML CI-derived CFU-GMs were cultured in liquid or semisolid media containing GM-CSF, they proliferated and differentiated along the neutrophil and macrophage lineages (Fig. 6F). However, the differentiation of many neutrophils was blocked at the promyelocyte stage. These blocked promyelocytes proliferated contin- uously as GM-CSF-dependent cell lines and were designated as EPRO /for EML-derived promyelocytes; see Materials and methods for derivation of these cell lines; Fig. 6G). Flow cytometry analyses of cell surface markers of a representative EPRO cell line $(\mathrm{Cl})$ show that $57 \%$ are positive for the neutrophil lineage-specific antigen $7 / 4$, few are positive for Sca-1, and none expresses B220 or Mac-1 (Fig. 7A). Southern analysis showed that EPRO Cl had the same proviral integration site as EML $\mathrm{Cl}$ and EML C1-derived B-cell precursors supported by stromal cell W20 and IL-7 (Fig. 7B), again verifying the common origin of these cells. The GM-CSF-dependent EPRO cell lines are virtually identical to the GM-CSF-dependent MPRO (mouse promyelocyte) cell lines that we isolated directly from LRAR $\alpha 403$ SN-infected, GM-CSF-stimulated mouse bone marrow as reported previously (Tsai and Collins 1993). Like MPRO cells, the EPRO cells differentiate synchronously and rapidly (in 96-120 hr) into mature neutrophils when treated with high concentrations of RA (Fig. $6 \mathrm{H}$ ). The optimal concentration for this induction of terminal differentiation is $0.5-1 \times 10^{-5} \mathrm{M}$ for EPRO cell line, similar to that for MPRO cells (Tsai and Collins 1993).

\section{Discussion}

In this paper we describe a unique, SCF-dependent lymphohematopoietic progenitor cell line (designated EML) with erythroid, myeloid, and lymphoid potentials. To our knowledge, the EML cell line is the only SCF-dependent cell line with both lymphoid and myeloerythroid potentials. It was established from a mouse bone marrow infected with a retroviral vector (LRAR $\alpha 403 S N$ ) harboring a dominant-negative RAR construct. Remarkably, all independently isolated EML-like cell lines harbor the LRAR $\alpha 403$ SN provirus despite the fact that the lymphohematopoietic progenitors represented only a small fraction of the input population and that no G418 selection was applied after retroviral infection, suggesting that lymphohematopoietic progenitors infected with the provirus possessed survival advantages over the uninfected cells. Furthermore, it appears that no common integration site was required to confer such survival advantages on the infected cells (Fig. 1C). Although the dominantnegative RAR $\alpha$ construct is essential for the establishment of EML-like cell lines, it is possible that additional mutations may have contributed to the emergence of the dominant clones.

The EML cell line is unique in its ability to generate large numbers of pre-pro-B cells. Furthermore, these prepro-B cells can respond to simultaneous stimulation with bone marrow stromal cells (W20) and IL-7 by expressing $R A G-1$ ) (Fig. 3B, lanes 10,11 ) and by initiating the rearrangement of the $D$ and $J$ segments of the IgH genes (Fig. 4B). The $D-I$ rearrangement occurs around day 8 , following the peak expression of RAG-1 on day 6 . We have not detected cytoplasmic or membrane $\mu$-chain protein by immunofluorescence in EML Cl cells stimulated with the W20 stromal cells and IL-7 for 10 days (not shown). It is of interest to determine if these pro-B cells 

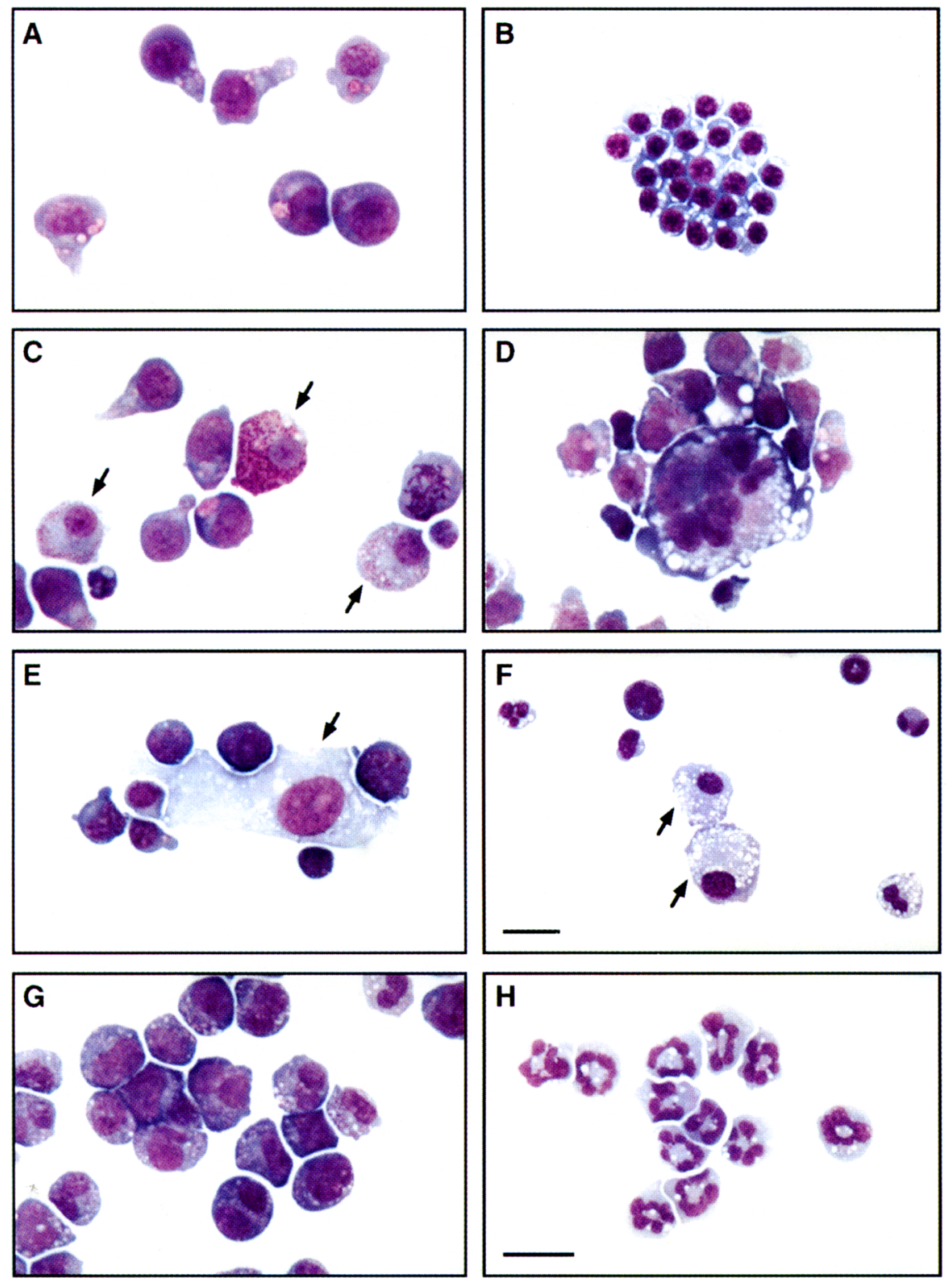

Figure 6. Erythroid, myeloid, and lymphoid cells developed from EML Cl. (A) EML Cl cells in growth medium (note the three hand-mirror-shaped cells that are frequently seen in the EML $\mathrm{Cl}$ cell line); $(B)$ a cluster of polychromatophilic erythroblasts; $|C|$ three coarsely granulated mast cells (arrows); (D) a megakaryocyte (center) with marked polyploidy; (E) developing B cells from a 14-day coculture of EML C1/W20/IL-7 [the large cell hugging the smaller B-cell precursors is a W20 stromal cell (arrow)]; $(F)$ vacuolated macrophages (arrows) surrounded by smaller neutrophils from an EML C1-derived CFU-GM colony; $(G)$ the neutrophilic promyelocyte cell line EPRO $\mathrm{Cl}$ derived from EML Cl (the cytoplasm contains numerous azurophilic granules); $(H)$ neutrophils with segmented nuclei that have developed from EPRO $\mathrm{Cl}$ treated with $10^{-5} \mathrm{M}$ RA for 5 days. Wright-Giemsa stain. All have the same magnifications except $F$. Bars, $50 \mu$. can continue their differentiation to the plasma cell stage and what stimuli are required for such processes.

The EML cell line also spontaneously generates cells recognized by the monoclonal antibody ter 119 , which recognizes mostly erythroid precursors (Ikuta et al. 1990). Three to five days after addition of Epo to the liquid culture of EML, we detected the appearance of hemoglobinized erythroblasts that formed aggregates of 4-32 cells (Fig. 6B). The rapidity of their appearance and their limited proliferative capacity suggest that these erythroblasts are the progenies of the so-called colonyforming units-erythroid (CFU-Es). In addition, large multicentric erythroid colonies containing several thousands of erythroblasts developing over 7-10 days could readily be demonstrated in clonal cultures of EML in methylcellulose medium supplemented with SCF and
Epo. These are derived from the so-called BFU-Es, which are developmentally more primitive than the CFU-Es and eventually differentiate into CFU-Es. Although flow cytometry indicated slightly more than $50 \%$ of EML C1 cells expressed the B-cell lineage-specific antigen B220 or the erythroid precursor-specific antigen ter 119 (Fig. 2), we believe this slight "overlapping" of the percentages of B220- and ter 119-positive cells resulted from minor imprecisions in setting fluorescence gates during data analysis and did not always occur.

In contrast with its spontaneous generation of large numbers of B-lymphoid and -erythroid progenitors, the SCF-dependent EML C1 cells produce very few progenitors for the neutrophil and macrophage lineages (CFUGMs; Table 1). This is in sharp contrast to the normal multipotent hematopoietic progenitors that readily gen- 
A

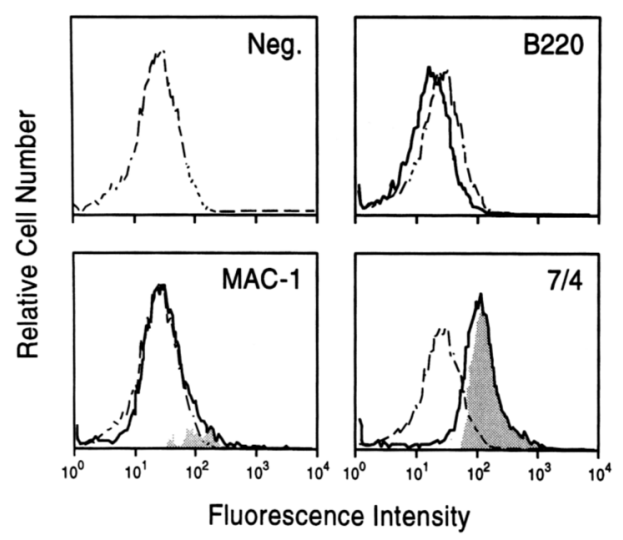

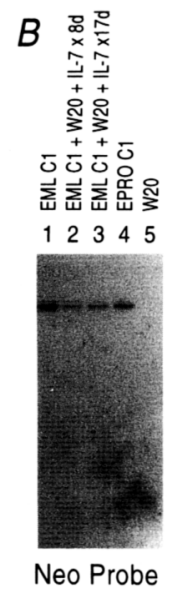

Figure 7. Phenotype and clonal origin of the EPRO cell line. $(A)$ Flow cytometry analyses of the cell surface antigens of EPRO $\mathrm{Cl}$. EPRO $\mathrm{Cl}$ is a GM-CSF-dependent neutrophilic promyelocyte cell line derived from EML as described in Materials and methods. The isotype antibody (negative control; thin line) profile is shown in the first panel and again in all other panels for direct comparison. The positive fractions (shaded areas) are indicated in each panel. Most cells in EPRO $\mathrm{Cl}$ are stained by neutrophil lineage-specific monoclonal antibody 7/4. $(B)$ Retroviral integration site of EPRO Cl. Genomic DNAs from EML C1 (lane 1), EML C1-derived B-cell precursors expanded on stromal cell line W20 plus IL-7 for 8 days (lane 2) or 17 days (lane 3), EPRO Cl (lane 4), and W20 alone (lane 5) were digested with EcoRI and analyzed as described in Fig. 1A. Samples in lanes 2 and 3 also contained DNAs of the W20 stromal cell line.

erate CFU-GMs in vitro. Intriguingly, this deficiency of CFU-GMs in EML Cl cells can be overcome with a combination of IL-3 and high concentrations of RA $\left(10^{-6}\right.$ $\left.10^{-5} \mathrm{M}\right)$, leading to a dramatic increase in the number of CFU-GMs (Table 1). (For comparison, the serum concentration of RA was estimated to be $10^{-9}-10^{-8} \mathrm{M}$; DeRuyter et al. 1979.) This phenomenon has never been described before but is in line with our previous observations that the expression of the dominant-negative RAR $\alpha 403$ in an IL-3-dependent multipotent hematopoietic cell line (FDCP mix A4) severely suppressed spontaneous neutrophil and macrophage development (Tsai et al. 1992) and that the dominant-negative effects of RAR $\alpha 403$ in myeloid cells could be overcome or bypassed by high concentrations $\left(10^{-6}-10^{-5} \mathrm{M}\right)$ of RA in both trans-activation and differentiation assays (Tsai and Collins 1993). Thus, it appears that the inhibition of CFU-GM formation in EML Cl is attributable to the activity of the dominant-negative RAR $\alpha 403$, and this inhibition can be overcome or bypassed by high concentrations of RA.

Further analyses revealed that the increase in CFUGMs was accompanied by a decrease in SCF-responsive, self-renewing clonogenic cells and in the total number of EML C1 cells after 3 or 4 days of treatment with SCF/ IL-3/RA (Table 1). These opposite trends, that is, a decrease in SCF-dependent self-renewal and an increase in
CFU-GMs, suggest that high concentrations of RA may have induced the multipotent progenitors in EML Cl cell line to commit to the neutrophil/macrophage differentiation pathway. As a result, the number of SCF-responsive, self-renewing clonogenic cells decreases, which in turn leads to a decrease in BFU-Es (Table 1, experiment 2). Although an additional, direct inhibitory effect of RA on self-renewing cells (as well as BFU-Es) cannot be ruled out, these observations raise the possibility that the immortalization of EML cells may be an immediate consequence of the suppressive effect of the dominant-negative RAR $\alpha 403$ on myeloid commitment. The primitive lymphohematopoietic progenitors are characterized by their ability to self-renew and to commit to several differentiation pathways. For primitive lymphohematopoietic progenitors to exist as a continuous cell line like EML, the probability of its self-renewal in vitro must exceed its probability of commitment to various differentiation pathways. By virtue of its ability to interfere with the progenitors' ability to commit to the myeloid differentiation pathway, the dominant-negative RAR $\alpha 403$ may have tipped the balance of self-renewal versus differentiation in favor of self-renewal, leading to the establishment of the EML cell line.

A second block in the differentiation of EML-derived neutrophil precursors occurs downstream at the promyelocyte stage, leading to the establishment of GM-CSFdependent promyelocyte cell lines such as EPRO (Fig. 6G). High concentrations of RA $\left(10^{-5}-10^{-6} \mathrm{M}\right)$ induce these cells to terminally differentiate into mature neutrophils (Fig. 6H). The biological properties (i.e., developmental stage, growth factor requirements, surface antigens, and RA responsiveness) of EPRO cells are virtually identical to those of GM-CSF-dependent MPRO cell lines that we previously established from LRAR $\alpha 403 \mathrm{SN}$ infected, GM-CSF-stimulated mouse bone marrow cells (Tsai and Collins 1993). Because we have shown that the RA-reversible differentiation block in MPRO cells is attributable to the dominant-negative effects of RAR $\alpha 403$ (Tsai and Collins 1993), we believe that the RA-reversible differentiation block in EPRO cells is also caused by the dominant-negative RAR $\alpha 403$.

In summary, our data indicate that RA and RARs play important roles in the development of neutrophils at two distinct stages in the EML Cl cell line: The first one is mapped to the pre-CFU-GM stage, whereas the second one occurs at the neutrophilic promyelocyte stage. Our findings suggest that RARs may be part of the combinatorial mix of transcriptional regulators that converge before the establishment of a "nodal decision point" (Lassar and Weintraub 1992) in myeloid lineage development. They also raise the possibility of influencing the self-renewal of lymphohematopoietic progenitors in vitro by adding strong antagonists of RA to the culture medium. The effects of the dominant-negative RAR $\alpha 403$ on myeloid lineage development in EML are summarized in Figure 8.

Because of the altered physiology brought on by the dominant-negative RAR, the EML cell line can not be equated with normal lymphohematopoietic progenitors. 


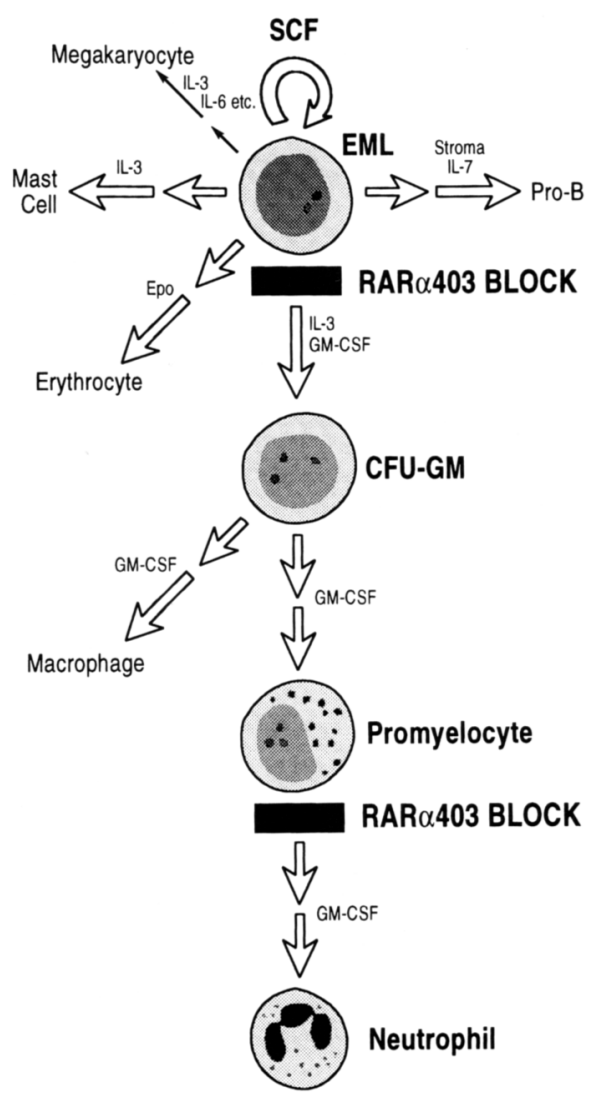

Figure 8. A schematic summary of the effects of the dominantnegative RAR $\alpha 403$ on myeloid development in the EML cell line. The SCF-dependent, self-renewing lymphohematopoietic progenitor in EML cell line is depicted at the top. The lineages that have been observed in the EML cell line are indicated. The developmental blocks imposed by the dominant-negative RAR (RAR $\alpha 403$ ) are mapped to the pre-CFU-GM and the neutrophilic promyelocyte stages. The block to CFU-GM formation may have increased the probability of self-renewal of the lymphohematopoietic progenitor. Both developmental blocks can be overcome by high concentrations $\left(0.1 \times 10^{5}\right.$ to $\left.1 \times 10^{-5} \mathrm{M}\right)$ of $\mathrm{RA}$, leading to the production of mature neutrophils and a decrease in the self-renewal of EML.

Nevertheless, it appears to be the closest in vitro approximation of the common lymphohematopoietic progenitor that has been established to date. As such, it provides a unique model system for studying the molecular control of early erythroid, myeloid, and lymphoid lineage development.

\section{Materials and methods}

\section{Bone marrow}

Six-week-old male $\mathrm{BDF}_{1}(\mathrm{C} 57 \mathrm{BL} / 6 \times \mathrm{DBA} / 2)$ (the Jackson Laboratory) were injected intraperitoneally with 5-fluorouracil (100 $\mathrm{mg} / \mathrm{kg}$ of body weight 5 days prior to bone marrow harvest. The low-density marrow cell fraction containing hematopoietic progenitors was collected by density centrifugation over Nycodenz (specific gravity, 1.080; GIBCO).

\section{Retroviral vectors}

The structure of the dominant-negative RAR $\alpha 403 \mathrm{cDNA}$, the construction of the retroviral vectors LXSN and LRAR $\alpha 403 S N$, and the establishment of helper virus-free amphotropic retroviral producer cell lines PA317/LXSN and PA317/LRAR $\alpha 403$ SN have been reported (Miller and Rosman 1989; Tsai et al. 1992). The viral titers of the supernatants of the producer cells average $1 \times 10^{6}$ to $5 \times 10^{6} / \mathrm{ml}$ as assayed on NIH-3T3 cells.

Retroviral infection and establishment of

Post-5-fluorouracil low-density $\mathrm{BDF}_{1}$ mouse bone marrow cells were infected by cocultivation with unirradiated, subconfluent amphotropic retroviral producer cells for 2 days in IMDM (Iscove's modified Dulbecco medium) supplemented with $20 \%$ \{vol/vol) horse serum, $20 \%$ WEHI $3 \mathrm{~B}$ conditioned medium [(vol/vol) as a source of murine IL-3], murine GM-CSF $(2.5 \mathrm{ng}$ / $\mathrm{ml}$; Immunex), human IL- $1 \beta$ ( $10 \mathrm{ng} / \mathrm{ml}$; Amgen), human IL-6 (20 $\mathrm{ng} / \mathrm{ml}$; Amgen), and polybrene $(4 \mu \mathrm{g} / \mathrm{ml})$. In the initial isolation of the EML cell line, only $10^{5}$ bone marrow mononuclear cells were used in the coculture infection; in later experiments $10^{6}$ cells were used. Nonadherent, infected cells were subsequently cultured in IMDM supplemented with $20 \%$ horse serum, rat SCF ( $200 \mathrm{ng} / \mathrm{ml}$; Amgen), 0.25\% WEHI-3B-conditioned medium (with the final concentration of IL-3 estimated to be 2.5-5.0 ng/ml), and human Epo (8 U/ml; Amgen). Cells were subcultured every 2-3 days. EML-like cells became the dominant cell type in 1 1/2-2 months. Although low concentrations of IL-3 were necessary in the establishment of the cell lines, higher concentrations of IL-3 promoted the growth of mast cells that seemed to retard the emergence of EML-like cells. Cell lines established from LRAR $\alpha 403 \mathrm{SN}$-infected cultures were maintained in IMDM supplemented with $20 \%$ horse serum and rat SCF ( $200 \mathrm{ng} / \mathrm{ml}$; Amgen) alone. Subcloning of the cell line was carried out first by limiting dilution at 0.4 clonogenic cells per well in 96-well plates. Lines obtained by limiting dilution cloning were recloned ( 300 cells per $35-\mathrm{mm}$ dish) in semisolid culture medium containing $0.8 \%$ methylcellulose plus SCF $(200$ $\mathrm{ng} / \mathrm{ml}$ ) once (EML C1) or twice (EML C1.1-C1.4). Individual colonies were picked with the aid of an inverted microscope and expanded in IMDM $/ 20 \%$ horse serum/SCF (200 ng/ml).

\section{Monoclonal antibodies}

Affinity-purified anti-B220 (also known as anti-Ly-5 or CD45R; clone RA3-6B2), anti-Mac-1, fluorescein-conjugated goat antirat $\operatorname{IgG}(\mathrm{H}+\mathrm{L})$ second antibody, anti-Sca-1, and rat $\operatorname{IgG}_{2 \mathrm{a}}$ isotype (control) antibody were purchased from PharMingen. Monoclonal anitbody ter 119 was generously provided by Tatsuo Kina (Kyoto University, Japan). Monoclonal antibody $7 / 4$ was a gift from Saimon Gordon (Oxford University, UK).

Induction of B-lymphoid, erythroid, and myeloid differentiation

The stromal cell line W20 was established from the bone marrow of a W $+1+1$ mouse and then subcloned four times (Tsai 1986). A clonal line W20 F1 was used in this experiment. It is adherent and has extensive, veil-like cytoplasm. Upon confluency, the cells undergo adipogenesis. It is maintained in Dulbecco's modified Eagle medium (DMEM) supplemented with $10 \%$ FCS. To induce B-cell differentiation, one million EML cells that have been washed twice with phosphate-buffered saline (PBS) to remove SCF were added to a confluent layer of W20 
grown in a 6-well plate and fed with RPMI 1640 supplemented with $5 \%$ FCS, $5 \times 10^{-5} \mathrm{M} 2$-mercaptoethanol, and human IL-7 (100 U/ml; Immunex) every $2-3$ days. Total RNAs and genomic DNAs were prepared from the stromal cell/EML cell cocultures without separation of the two cell types.

For induction of erythroid differentiation in liquid culture, EML cells were cultured in IMDM supplemented with $20 \%$ horse serum, SCF $(200 \mathrm{ng} / \mathrm{ml})$, and Epo $(8 \mathrm{U} / \mathrm{ml})$. Benzidine (Sigma) staining was performed as described (Orkin et al. 1975). For BFU-E assay, EML cells were cultured in $0.8 \%$ methylcellulose culture medium supplemented with $20 \%$ horse serum, $\mathrm{SCF}(200 \mathrm{ng} / \mathrm{ml})$, and Epo $(8 \mathrm{U} / \mathrm{ml})$.

For induction of CFU-GM, the EML cells were cultured in IMDM supplemented with $20 \%$ horse serum, rat SCF $(200 \mathrm{ng} /$ mi) plus $5 \%$ WEHI conditioned medium. Cultures were treated with all-trans RA at various concentrations for 72-96 hr. Cells were then washed three times with PBS to remove RA and recultured in IMDM/methylcellulose $(0.8 \%)$ supplemented with $20 \%$ horse serum and murine GM-CSF $(10 \mathrm{ng} / \mathrm{ml})$ for CFU-GM assay, SCF (200 ng/ml) for detecting self-renewing clonogenic cells, or SCF plus Epo $(8 \mathrm{U} / \mathrm{ml})$ for BFU-E assay.

\section{Establishment of neutrophilic promyelocyte cell lines (EPRO) from EML}

EML C1 cells induced with RA plus IL-3 (described above) were washed to remove exogenous RA and then cultured directly in liquid medium (IMDM $/ 20 \%$ horse serum) plus GM-CSF (10 ng/ ml). Most cells died upon shifting to GM-CSF, whereas CFUGMs proliferated and differentiated into neutrophils, promyelocytes, and macrophages. After 2-3 weeks, most of the growing cells were promyelocytes that depended on GM-CSF $(10 \mathrm{ng} / \mathrm{ml})$ for survival and proliferation. Alternatively, individual CFUGM colonies (described above) were picked from the methylcellulose culture and expanded in liquid medium containing GMCSF. Subcloning was done in methylcellulose supplemented with $20 \%$ horse serum and GM-CSF $(10 \mathrm{ng} / \mathrm{ml})$.

\section{Southern and Northern analyses}

For Southern analysis of the number and sites of proviral integrants, genomic DNA samples were isolated from various cell lines and digested with EcoRI, electrophoresed ( $25 \mu \mathrm{g}$ per lane), blotted onto nitrocellulose, and hybridized with a ${ }^{32} \mathrm{P}$ nicktranslated $0.9-\mathrm{kb}$ neo probe. The correct size of the provirus within all cell lines was also verified by digestion with SmaI (which cut only within the long terminal repeats of the provirus) and analyzed by Southern analysis using neo as the probe (not shown). Northern blots of total RNAs from various cell lines and tissues were hybridized with the following nick-translated probes: a 0.3-kb SalI-KpnI fragment of immunoglobulin $\mathrm{C}_{\mu 3}$ genomic DNA clone encoding the third exon of the mouse IgH constant region gene (provided by Gregory Warr, Medical University of South Carolina, Charleston); a $0.95-\mathrm{kb}$ BgIII fragment containing part of the coding region of a mouse $R A G-1$ genomic clone (provided by Roger Perlmutter and Steve Anderson, University of Washington, Seattle, WA); a mouse $\beta^{\text {major }}$ globin cDNA probe (obtained from Mark Groudine, Fred Hutchinson Cancer Center, Seattle, WA), and a 226-bp fragment of the $J_{3}$ segment of mouse IgH gene prepared as described below.

\section{PCR detection of $\mathrm{D}-\mathrm{J}$ rearrangement}

The primers for PCR detection of $D-I$ rearrangements were DHL, GMTTTTTGTSAAGGGATCTACTACTGTG and J3, TTCTCACAAGAGTCCGATAGACCCTGG; $15^{\prime} \rightarrow 3^{\prime} ; \quad M=A$ or $C ; S=C$ or $G$ ). Each 100- $\mu$ 1 PCR reaction contained $0.2 \mu \mathrm{g}$ of spleen genomic DNA or $2 \mu \mathrm{g}$ of test template DNAs, $10 \mathrm{~mm}$ Tris (pH 8.3 at room temperature), $50 \mathrm{mM} \mathrm{KCl}, 1.5 \mathrm{~mm} \mathrm{MgCl}_{2}$, $0.01 \%$ gelatin, $100 \mathrm{ng}$ of each primer, and 1 unit of Taq polymerase (Amplitaq; Cetus). Thirty cycles of amplifications were performed. Each cycle consisted of $94^{\circ} \mathrm{C}$ for $1 \mathrm{~min}, 60^{\circ} \mathrm{C}$ for 1 min, and $72^{\circ} \mathrm{C}$ for $2 \mathrm{~min}$, followed by a single $10 \mathrm{~min}$ at $72^{\circ} \mathrm{C}$. Twelve microliters of PCR products were electrophoresed in $1 \%$ agarose, blotted onto nitrocellulose membranes, and probed with a nick-translated $226-\mathrm{bp} I_{3}$ probe. The $I_{3}$ probe was prepared by PCR amplification of the spleen DNA of a $\mathrm{BDF}_{1}$ mouse using DHL and J3 primers, followed by TA cloning of the $\sim 319$ bp PCR product into the pT7Blue vector (Novagene). The sequence of the $\sim 319$-bp PCR product was verified as those of $\mathrm{D}-I_{3}$ by Taq DyeDeoxy Terminator Cycle Sequencing (Applied Biosystems) using the DHL and 13 primers. The $\mathrm{pT} 7-I_{3}$ plasmid was digested with HindIII and StyI and the 226-bp fragment containing the $J_{3}$ sequence internal to the DHL and J3 primers was gel-purified and nick-translated before hybridization.

\section{Acknowledgments}

We wish to thank Mark Groudine, Eric Milner, Irv Bernstein, and Jerry Radich for helpful discussions and Hal Weintraub for critical comments on this manuscript. Many thanks go to LeMoyne Mueller and Paula Ladne for excellent technical assistance. This work was supported by National Cancer Institute grants CA01676 (S.T.) and CA58292 (S.J.C.).

The publication costs of this article were defrayed in part by payment of page charges. This article must therefore be hereby marked "advertisement" in accordance with 18 USC section 1734 solely to indicate this fact.

\section{References}

Alcalay, M., D. Zangrilli, P. Pandolfi, L. Longo, A. Mencarelli, A. Giacomucci, M. Rocchi, A. Biondi, A. Rambaldi, F. Lo Coco, D. Diverio, D. Donti, E. Donti, F. Grignani, and P. Pelicci. 1991. Translocation breakpoint of acute promyelocytic leukemia lies within the retinoic acid receptor $\alpha$ locus. Proc. Natl. Acad. Sci. 88: 1977-1981.

Alt, F., T. Blackwell, and G. Yancopoulos. 1987. Development of the primary antibody repertoire. Science 238: 1079-1087.

Borrow, J., A.D. Goddard, D. Sheer, and E. Solomon. 1990. Molecular analysis of acute promyelocytic leukemia breakpoints cluster region on chromosome 17. Science 249: 15771580.

Breitman, T.R., S.E. Selonick, and S.J. Collins. 1980. Induction of differentiation of the human promyelocytic leukemia cell line (HL-60) by retinoic acid. Proc. Natl. Acad. Sci. 77: 29362940.

Coffman, R.L. and I.L. Weissman. 1981. B220: A B cell-specific member of the T200 glycoprotein family. Nature 289: 681683.

Collins, S.J., K. Robertson, and L. Mueller. 1990. Retinoic acidinduced granulocytic differentiation of HL-60 myeloid leukemia cells is mediated directly through the retinoic acid receptor (RAR $\alpha$ ). Mol. Cell. Biol. 10: 2154-2161.

Damm, K., R.A. Heyman, K. Umesono, and R.M. Evans. 1993. Functional inhibition of retinoic acid response by dominant negative retinoic acid receptor mutants. Proc. Natl. Acad. Sci. 90: 1989-2993.

DeRuyter, M.G., W. Lambert, and P. DeLunheer. 1979. Retinoic acid: An endogenous compound of human blood. Unequivocal demonstration of endogenous retinoic acid in normal 
physiological conditions. Anal. Biochem. 98: 402-409.

De The, H., C. Chomienne, M. Lanotte, L. Degos, and A. Dejean. 1990. The $t(15 ; 17)$ translocation of acute promyelocytic leukemia fuses the retinoic acid receptor $\alpha$ gene to a novel transcribed locus. Nature 347: 558-561.

de The, H., C. Lavau, A. Marchio, C. Chomienne, L. Degos, and A. Dejean. 1991. The PML-RAR $\alpha$ fusion mRNA generated by the $t(15 ; 17)$ translocation in acute promyelocytic leukemia encodes a functionally altered RAR. Cell 66: 675-684.

Dyck, J.A., G.G. Maul, W.H. Miller Jr., J.D. Chen, A. Kakizuka, and R.M. Evans. 1994. A novel macromolecular structure is a target of the promyelocyte-retinoic acid receptor oncoprotein. Cell 76: 333-343.

Evans, R.M. 1988. The steroid and thyroid hormone receptor superfamily. Science 240: 889-895.

Hardy, R.R., C.E. Carmack, S.A. Shinto, J.D. Kemp, and K. Hayakawa. 1991. Resolution and characterization of pro-B and pre-pro-B cell stages in normal mouse bone marrow. $/$. Exp. Med. 173: 1213-1225.

Hirsch, S. and S. Gordon. 1983. Polymorphic expression of a neutrophil differentiation antigen revealed by monoclonal antibody 7/4. Immunogenetics 18: 229-239.

Huang, M.-E., Y.-C. Ye, S.-R. Chen, J.-R. Chai, J.-X. Lu, L. Zhoa, H.T. Gu, and Z.-Y Wang. 1988. Use of all-trans retinoic acid in the treatment of acute promyelocytic leukemia. Blood 72: $567-572$.

Ikuta, K., T. Kina, I. MacNeil, N. Uchida, B. Peault, Y.-H. Chien, and I.L. Weissman. 1990. A developmental switch in thymic lymphocyte maturation potential occurs at the level of hematopoietic stem cells. Cell 62: 863-874.

Kakizuka, R., W.H. Miller Jr., K. Umesono, R.P. Warrell, S.R. Frankel, V.V.V.S. Murty, E. Dmitrovsky, and R.M. Evans. 1991. Chromosomal translocation $t(15 ; 17)$ in human acute promyelocytic leukemia fuses RAR $\alpha$ with a novel putative transcription factor, PML. Cell 66: 663-674.

Kierney, P.C. and K. Dorshkind. 1987. B lymphocyte precursors and myeloid progenitors survive in diffusion chamber culture but $B$ cell differentiation requires close association with stromal cells. Blood 70: 1418-1424.

Lassar, A.B. and H. Weintraub. 1992. The myogenic helix-loophelix family: Regulators of skeletal muscle determination and differentiation. In Transcriptional regulation (ed. S.L. McKnight and K.R. Yamamoto), pp. 1037-1061. Cold Spring Harbor Laboratory Press, Cold Spring Harbor, New York.

Lemischka, I.R., D.H. Raulet, and R.C. Mulligan. 1986. Developmental potential and behavior of hematopoietic stem cells. Cell 45: 917-927.

Metcalf, D. 1993. Hematopoietic regulators: Redundancy or subtlety? Blood 82: 3515-3523.

Miller, A.D. and G.J. Rosman. 1989. Improved retroviral vectors for gene transfer and expression. BioTechniques 7: 980-990.

Nagpal, S., M. Saunders, P. Kastner, B. Durand, H. Nakshatri, and P. Chambon. 1992. Promoter context- and response element-dependent specificity of the transcriptional activation and modulating functions of retinoic acid receptors. Cell 70: 1007-1019.

Nelson, K.J., J. Haimovich, and R.P. Perry. 1983. Characterization of productive and sterile transcripts from the immunoglobulin heavy-chain locus: Processing of $\mu_{\mathrm{m}}$ and $\mu_{\mathrm{s}}$ mRNA. Mol. Cell. Biol. 3: 1317-1332.

Orkin, S.H., F. Harosi, and P. Leder. 1975. Differentiation in erythroleukemic cells and their somatic hybrids. Proc. Natl. Acad. Sci. 72: 98-102.

Schatz, D.G., M.A. Oettinger, and D. Baltimore. 1989. The V(D)J recombination activating gene, RAG-1. Cell 59: 1035-1048.

Schlissel, M.S. and D. Baltimore. 1989. Activation of immuno- globulin kappa gene rearrangement correlates with induction of germline kappa gene transcription. Cell 8: 10011007.

Schlissel, M.S., L.M. Corcoran, and D. Baltimore. 1991. Virustransformed pre-B cells show ordered activation but not inactivation of immunoglobulin gene rearrangement and transcription. J. Exp. Med. 173: 711-720.

Spangrude, G.J., S. Heimfeld, and I.L. Weissman. 1988. Purification and characterization of mouse hematopoietic stem cells. Science 241: 58-62.

Thomas, M.L. 1989. The leukocyte common antigen family. Annu. Rev. Immunol. 7: 339-369.

Tsai, S. 1986. "The functional roles of stromal cells in erythropoiesis." Ph.D. thesis, Harvard University, Cambridge, MA.

Tsai, S. and S.J. Collins. 1993. A dominant-negative retinoic acid receptor blocks neutrophil differentiation at the promyelocyte stage. Proc. Nat1. Acad. Sci. 90: 7153-7157.

Tsai, S., S. Bartelmez, R. Heyman, K. Damm, R. Evans, and S.J. Collins. 1992. A mutated retinoic acid receptor- $\alpha$ exhibiting dominant-negative activity alters the lineage development of a multipotent hematopoietic cell line. Genes \& Dev. 6: 2258-2269.

Weis, K., S. Rambaud, C. Lavau, J. Jansen, T. Carvalho, M. Carmo-Fonseca, A. Lamond, and A. Dejean. 1994. Retinoic acid regulates aberrant nuclear localization of PML-RAR $\alpha$ in acute promyelocytic leukemia cells. Cell 76: 345-356.

Wu, A.M., J.E. Till, L. Siminovitch, and E.A. McCulloch. 1968. Cytological evidence for a relationship between normal hematopoietic colony-forming cells and cells of lymphoid system. J. Exp. Med. 127: 455-464.

Zsebo, K.M., J. Wypych, I.K. McNeice, H.S. Lu, K.A. Smith, S.B. Karkare, R.K. Sachdev, V.N. Yuschenkoff, N.C. Birkett, L.R. Williams, V.N. Satyagal, W. Tung, R.A. Bosselman, E.A. Mendiaz, and K.E. Langley. 1990. Identification, purification, and biological characterization of hematopoietic stem cell factor from Buffalo rat liver-conditioned medium. Cell 63: 195-201. 


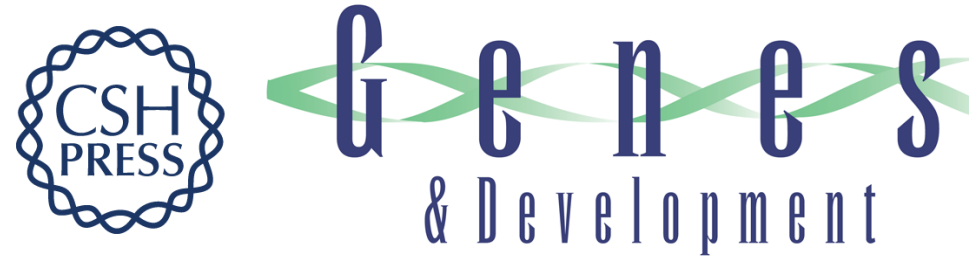

\section{Lymphohematopoietic progenitors immortalized by a retroviral vector harboring a dominant-negative retinoic acid receptor can recapitulate lymphoid, myeloid, and erythroid development.}

S Tsai, S Bartelmez, E Sitnicka, et al.

Genes Dev. 1994, 8:

Access the most recent version at doi:10.1101/gad.8.23.2831

References This article cites 34 articles, 17 of which can be accessed free at:

http://genesdev.cshlp.org/content/8/23/2831.full.html\#ref-list-1

License

Email Alerting Service

Receive free email alerts when new articles cite this article - sign up in the box at the top right corner of the article or click here.

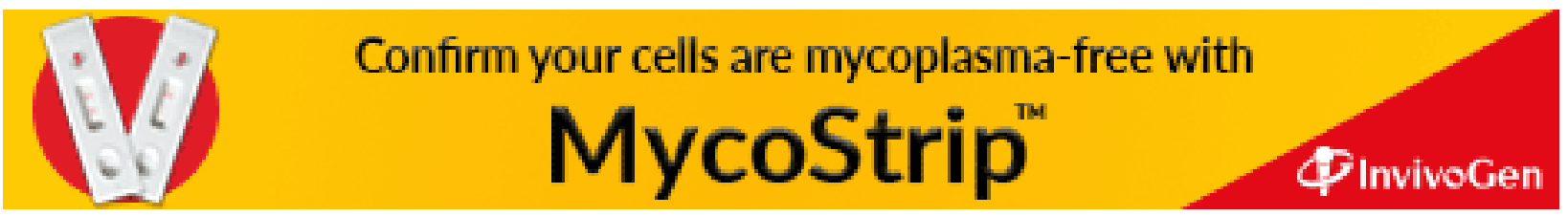

